\title{
Cytokine production in ex-vivo stimulated fresh and cryopreserved T-cells
}

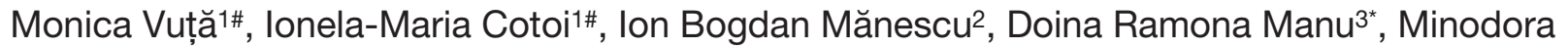 \\ Dobreanu ${ }^{1,2,3}$ \\ 1. George Emil Palade University of Medicine, Pharmacy, Science, and Technology of Targu Mures, Romania \\ 2. County Emergency Clinical Hospital of Targu Mureș, Romania \\ 3. Center for Advanced Medical and Pharmaceutical Research, Targu-Mures, Romania
}

\begin{abstract}
Objective: In vitro cytokine production by peripheral blood mononuclear cells (PBMCs) is an important and reliable measure of immunocompetence. PBMC can be stimulated directly after isolation or frozen for later use. However, cryopreservation may affect cell recovery, viability and functionality. This study aims to investigate cytokine synthesis in ex-vivo stimulated fresh and cryopreserved CD4+ and CD4- T cells. Methods: PBMCs were obtained by Ficoll gradient centrifugation from heparinized peripheral blood of 6 middle-aged clinically healthy subjects. Half of these cells (labeled "Fresh") was further processed and the other half (labeled "Cryo") was cryopreserved at $-140^{\circ} \mathrm{C}$ for up to 3 months. Fresh-PBMCs were activated with Phorbol-Myristate-Acetate/lonomycin/Monensin for 5 hours immediately after isolation while CryoPBMCs were identically activated after thawing and cell resting. Activated cells were fixed, permeabilized and intracellular cytokine staining was performed using Phycoerythrin (PE)-conjugated antibodies for Interleukin-2 (IL-2), Tumor Necrosis Factor-alpha (TNF-a), and Interferongamma (IFN-g). All samples were analyzed within 24 hours by flow cytometry. Results: Both Fresh and Cryo CD3+CD4+/CD3+CD4- subpopulations partially produced each of the three cytokines. A higher percentage of CD4+ T cells produced IL-2 and TNF-a and a greater percentage of CD4- T cells were found to produce IFN-g. A significantly higher percentage of Cryo-lymphocytes was shown to produce TNF-a in both $\mathrm{CD} 3+\mathrm{CD} 4+(31.4 \%$ vs $24.9 \%, \mathrm{p}=0.031)$ and $\mathrm{CD} 3+\mathrm{CD} 4-(22.7 \%$ vs $17.9 \%, \mathrm{p}=0.031)$ subpopulations. No notable difference was found for IL-2 and IFN-g production between Fresh and Cryo T cells. Conclusion: Cryopreservation for up to 3 months significantly increases TNF-a production of T-cells in clinically healthy middle-aged subjects.
\end{abstract}

Keywords: stimulated T cells, PBMC, cryopreservation, cytokine, intracellular cytokine staining

Received 22 February 2021 / Accepted 15 March 2021

\section{Introduction}

Peripheral blood mononuclear cells (PBMC) include immune cell types such as lymphocytes and monocytes. These cells can be efficiently obtained by Ficoll density gradient centrifugation from whole blood. When assessing the immune system, PBMCs are arguably the most studied cells due to their availability and key roles in immunity.

PBMCs are able to synthetize and secrete various cytokines. These signaling molecules are important mediators of immune and inflammatory responses, growth, differentiation and apoptosis of various cells [1]. Cytokine production tends to be limited in space and time as cytokine molecules typically act in an autocrine and paracrine manner, having a very short half-life [2]. Thus, in vivo assessment of cytokine production becomes a challenging task, driving scientists to find alternative methods for the study of cytokines. Flow cytometry analysis of cytokine synthesis is an elaborate technique which requires cell stimulation followed by intracellular cytokine staining (ICS), but has the advantage of individual cell analysis and phenotyping of the cytokine producing cells [3-6]. ICS protocols require cell fixation and permeabilization in order to allow the fluorochrome-conjugated antibodies to enter the cell. This step is necessary because cytokines are retained within

* Correspondence to: Doina Ramona Manu. E-mail: doina.manu@umfst.ro

\# Authors with equal contribution to this study the cell due to the use of intracellular protein transport inhibitors such as Monensin (MON) or Brefeldin A in the stimulation process. This determines the accumulation of cytokines into the cell in sufficient concentration to emit detectable fluorescent signals. ICS is a valuable assay for vaccine development $[4,7,8]$ and for the study of autoimmune diseases [9] and cancer [10].

PBMCs can be used as fresh cells or cryopreserved for later various structural and functional studies. Since large cohort studies frequently require sample cryopreservation, the use of cryopreserved PBMCs gained popularity over the years. The process of cryopreserving cells consists of maintaining cells in viable but dormant state at deeply low temperatures using cryoprotective agents like dimethyl sulfoxide (DMSO), glycerol and 1,2-propanediol $[11,12]$. This is challenging because, besides the need for monitoring the quality of frozen cells [13], there is a concern regarding the differences in gene expression and functionality (e.g. cytokine production) that might come with cryopreservation and thawing of cells [14-16].

The aim of this study was to observe and compare cytokine production between ex-vivo stimulated $\mathrm{T}$ cells from freshly isolated versus cryopreserved PBMC. The cytokines of interest were interleukin-2 (IL-2), tumor necrosis factor-alpha (TNF- $\alpha$ ) and interferon-gamma (IFN- $\gamma)$. 


\section{Materials and methods Blood samples}

Peripheral blood samples were collected from 6 healthy donors ( 3 men and 3 women) aged 40-50 years (mean age of 48) after overnight fasting. For each subject, two blood samples in $9 \mathrm{~mL} \mathrm{NH}$ Sodium Heparin vacutainer tubes (Greiner Bio-One, Cat. No. 455051) were collected. All participants gave written, informed consent and the study has been organized with the approval of the ethics committee of the "George Emil Palade" University of Medicine, Pharmacy, Science and Technology (GEP UMPhST) from Târgu-Mureș, Romania (decision No. $165 / 6^{\text {th }}$ of June 2019). Sample collection and processing were performed at the Advanced Medical and Pharmaceutical Research Center (CCAMF) of GEP UMPhST.

\section{PBMC isolation}

Prior to isolation, a complete blood count (CBC) was performed using the Sysmex XS-800i automatic hematology analyzer and C-reactive protein (hsCRP) plasma level was measured by nephelometry (BN ProSpec, Siemens).

Blood samples were processed within $2 \mathrm{hrs}$ from collection. PBMCs were isolated using a high-yield density gradient centrifugation protocol that was already tested and implemented in our laboratory [17]. In between the centrifugation steps, cells were resuspended in complete RPMI medium (cRPMI) consisting of: RPMI 1640 culture medium Sigma Aldrich (Cat. No. R8758) supplemented with 10\% Fetal Bovine Serum (FBS Sigma Aldrich, Cat. No. F7524) and 1\% Antibiotic Antimycotic Solution (Sigma Aldrich, Cat. No. A5955). The freshly isolated PBMCs were also suspended in RPMI and counted. Half of these cells (labeled "Fresh") were further processed for activation while the other half (labeled "Cryo") were cryopreserved for future activation.

\section{Cryopreservation and thawing}

Cryo-PBMCs were placed in RPMI 1640 culture medium supplemented with $20 \%$ FBS and 10\% Dimethyl sulfoxide (DMSO, Sigma Aldrich, Cat. No. D2438), stored overnight at $-80^{\circ} \mathrm{C}$ in a FTS30 freezing container (Corning, Cat. No. 432007) and then transferred at $-140^{\circ} \mathrm{C}$ for up to 3 months.

Thawing was done rapidly in a pre-heated $37^{\circ} \mathrm{C}$ water bath and cells were washed with cRPMI medium and centrifuged. After discarding the supernatant, cells were resuspended in cRPMI and incubated for resting in a 5\%, $100 \%$ humidity incubator at $37^{\circ} \mathrm{C}$ for $2 \mathrm{~h}$. After incubation, cells were re-washed and prepared for activation.

\section{PBMC activation}

For cell activation, Phorbol 12-Myristate 13-Acetate (PMA, $50 \mathrm{ng} / \mathrm{mL}$, Sigma Aldrich, Cat. No. P8139), Ionomycin (ION $1 \mu \mathrm{g} / \mathrm{mL}$, Sigma Aldrich, Cat. No. I0634) and BD GolgiStop (containing Monensin, MON $1.5 \mu \mathrm{L} / \mathrm{mL}$, Cat. No. 554724) were used. Both Fresh- and Cryo-PBMCs were activated in triplicate alongside control (CTRL), unstimulated cells. After 5 hours of incubation at $37^{\circ} \mathrm{C}$, cells were washed with phosphate buffered saline (PBS) and centrifuged at $300 \times \mathrm{g}$ for 5 minutes at $10^{\circ} \mathrm{C}$ without brake. Cell pellets were resuspended in Stain Buffer (BD Pharmingen, Cat. No. 554656) and cell suspensions were adjusted to a concentration of $10^{6}$ cells $/ \mathrm{mL}$.

\section{Cell fixation and staining}

Surface staining was performed using anti-human CD3Alexa-Fluor700 (BD Pharmingen, Cat. No. 557943) and CD4-PerCP-Cy5.5 (BD Pharmingen, Cat. No. 560650) antibodies, $5 \mu \mathrm{L}$ each $/ 10^{6}$ cells, followed by incubation on ice for 10 minutes in the dark.

For cell fixation, the activated PBMCs were suspended in Fixation/Permeabilization solution for 20 minutes. In order to maintain cell membrane permeability, Perm/Wash buffer was used for the following steps of the protocol (Fixation/Permeabilization Solution Kit, BD Biosciences, Cat. No. 554714).

For intracellular staining, we added $20 \mu \mathrm{L} / 10^{6}$ cells of each of the following PE-conjugated anti-human antibodies: 1:1 cocktail of Rat IgG2b $\kappa$ (BD Pharmingen, Cat. No. 553989) and Mouse IgG1 $\kappa$ (BD Pharmingen, Cat. No. 559320) for isotype controls; anti-IL-2 (BD Pharmingen, Cat. No. 554566); anti-TNF- $\alpha$ (BD Pharmingen, Cat. No. 559321) and anti-IFN- $\gamma$ (BD Pharmingen, Cat. No. 559327) respectively. This step was followed by incubation for 15 minutes at RT in the dark. Cells were washed $1 \times$ with Perm/Wash and 1× with Stain Buffer.

\section{Cell viability assessment}

For viability testing, the Annexin-V/Propidium iodide double staining method was used, following the kit's instructions (FITC Annexin V Apoptosis Detection Kit I, BD Pharmingen Cat. No. 556547). Cell viability was assessed at two time points throughout the protocol: postisolation and post-activation. For Cryo-cells additional viability assessments were performed post-thaw and postrest.

\section{Analysis and data processing}

Viability was analyzed ad-hoc during the protocol. ICS samples were analyzed within $24 \mathrm{hrs}$ from activation. All samples were analyzed on the same FACSAria III Cytometer using FACSDiva Software v8.0 (both from BD Biosciences). Instrument calibration was done using $\mathrm{BD}$ setup and tracking beads (BD Biosciences, Cat. No. 655050). BD FACSAria III cytometer optics used for data acquisition were described elsewhere [18]. Window extension was set at $2 \mu$ s, sheath pressure at 70 psi and a 70 -micron nozzle was used. For viability assessment, 10000 events were collected for each sample and PBMCs were gated based on their size and granularity (FSC/SSC) into two main populations: lymphocytes and monocytes. Viability assessment was performed on gated lymphocyte singlets as follows: 
double negative for viability, Annexin- $V$ single positive for apoptosis and double positive for late apoptosis/necrosis. For the ICS samples, a minimum of 25000 events in the lymphocyte gate were collected for each sample, all CD3+ singlets were gated into two populations $(\mathrm{CD} 3+\mathrm{CD} 4+$ and $\mathrm{CD} 3+\mathrm{CD} 4-)$ and the percentages (\%) of cytokine-producing cells were determined for each.

The non-parametric Wilcoxon signed rank test was used to compare paired data generated from fresh and frozen PBMC and $\mathrm{p}$-values $<0.05$ were considered significant. All statistical processing was performed using MedCalc v14.8 software. Data charts were generated in MedCalc v14.8 software and flow cytometry plots were generated in FlowJo 10 software.

\section{Results}

Surface staining and ICS were performed for all subjects $(n=6)$ and cells were analyzed by flow cytometry using the gating strategy described in Figure 1.

\section{CD3+ subset distribution}

For Fresh-PBMC, the CD3+ singlets were 47.6\% CD4+ ( $41.2 \%$ to $64.1 \%)$ and $32.5 \%$ CD4- (23.1\% to $35.7 \%)$, accounting for a mean $\mathrm{CD} 3+\mathrm{CD} 4+: \mathrm{CD} 3+\mathrm{CD} 4-$ ratio of 1.72 (1.15 to 2.59). For Cryo-PBMC, 54.9\% of the CD3+ singlets were $\mathrm{CD} 4+(50.8 \%$ to $57.4 \%)$ and $24.2 \%$ were CD4- (22\% to $29.8 \%)$ with a CD3+CD4+: CD3+CD4mean ratio of 2.25 (1.9 to 2.6). All reported values except ratios are median percentage values of the parent popu-
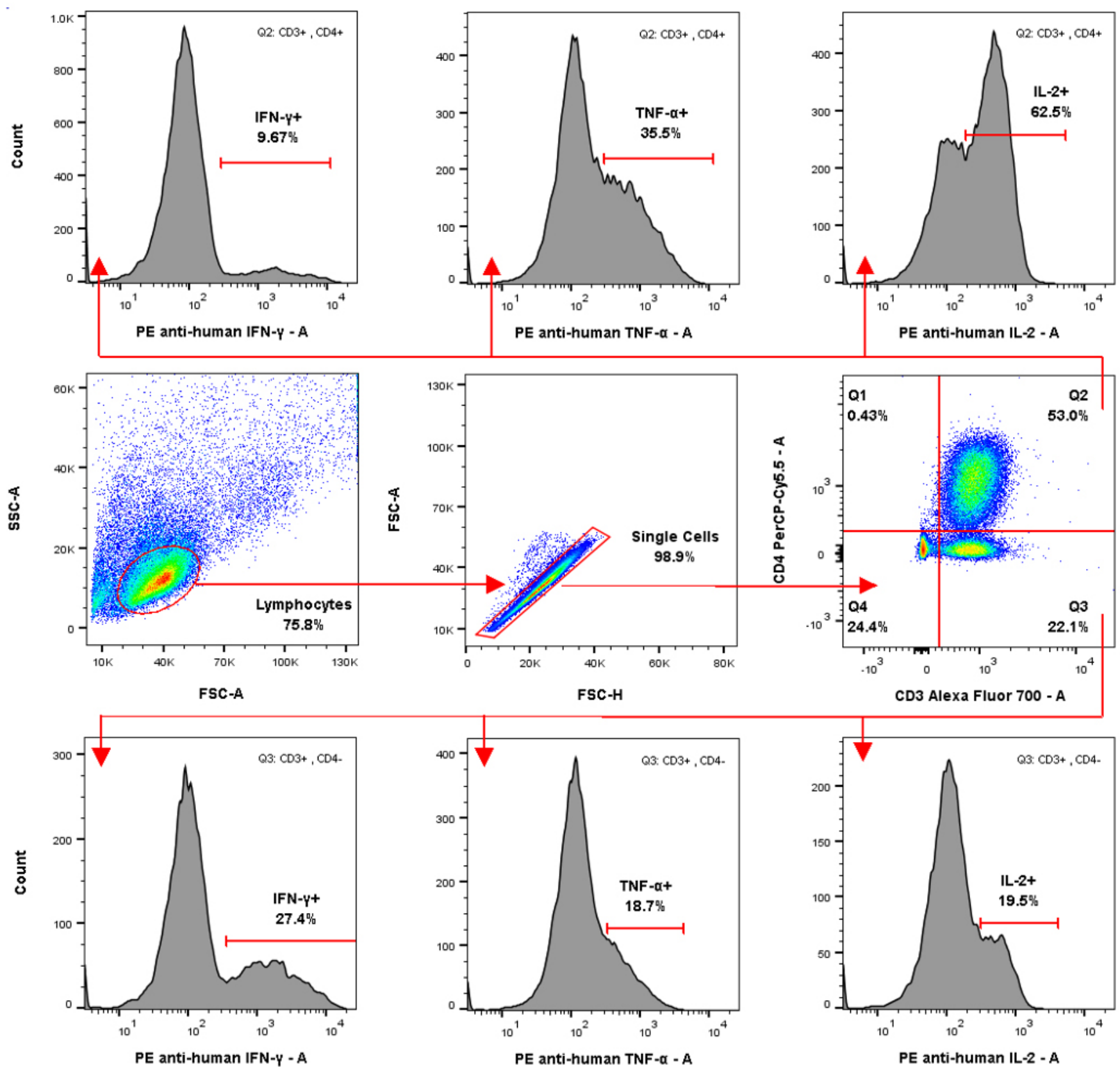

Fig. 1. Flow cytometry gating strategy. (From left to right) middle - pseudocolor density plots: identifying the lymphocyte population; doublet discrimination; gating of the CD4+ and CD4- cells from the CD3+ population; upper- histograms showing the cytokine producing CD3+CD4+ cells (IFN- $\gamma$, TNF- $\alpha$, IL-2); lower - histograms showing the cytokine producing CD3+CD4- cells (IFN- $\gamma$, TNF- $\alpha$, IL-2). 
lation (CD3+). No significant differences were observed between Fresh- and Cryo-PBMC regarding CD3+ subset distribution.

\section{Cytokine-producing cells}

Cytokine production in activated PBMC was compared between Fresh- and Cryo-cells for both subsets of the CD3+ population and data are presented in Table I. Generally, a significantly higher percentage of CD $4+$ comparatively with CD4- cells, produce IL-2 (Fresh $\mathrm{p}=0.03$, Cryo $\mathrm{p}=0.03)$. A higher, but not significant percentage of CD4+ cells produce TNF- $\alpha$ (Fresh $\mathrm{p}=0.15$, Cryo $\mathrm{p}=0.06)$. On the contrary, a greater percentage of CD4- comparatively with CD4+ cells, were found to produce IFN- $\gamma$ (Fresh $\mathrm{p}=0.03$, Cryo $\mathrm{p}=0.03)$. No cytokines of interest were detected in CTRL cells.

Regarding the effect of storage, cryopreservation did not affect the proportion of T-cells producing IL-2 and IFN- $\gamma$ after PMA stimulation. However, cryopreservation seems to significantly increase the percentage of TNF $\alpha$-producing T-cells.

\section{Viability}

As reflected in the median viability values, PMA activation of PBMC significantly reduced viability in both Fresh (53.2\% vs CTRL $87.3 \%)$ and Cryo cells (69.5\% vs CTRL $85.2 \%$ ) (Figure 2). The median viability of activated Cryo cells was apparently higher than for Fresh cells (69.5\% vs $53.2 \%)$, but the difference was not statistically significant $(\mathrm{p}=0.093)$. Upon thawing, the median viability of cryo- preserved cells dropped significantly from $88.9 \%$ (postisolation) to $67.1 \%$, but no significant changes occurred during the 2 hours of resting $(63.2 \%)$.

\section{Discussions}

\section{Cell viability - thawing, resting and stimulation}

In our study, viability of cryopreserved PBMC dropped significantly comparatively with fresh cells but no significant changes occurred during the 2 hours of resting before activation. PMA activation also significantly reduced viability in both fresh and cryopreserved cells.

Cell loss occurred at each step of our experiment, mainly during cell freezing/thawing, washing and especially during cell stimulation. The reason why substantially fewer than expected viable cells were recovered in some of our subjects is not known, but most likely relates in part to variations in protocol with regard to the number of cells originally placed in the cryovials. Wang et al. demonstrated that the duration of cryopreservation does not influence cell survival, but that cell loss is more likely linked to the process of cryopreservation [19]. A multicenter study suggested that cryopreserved cells can be stored for at least 12 years with no general tendency toward cell loss over time. Furthermore, there was no statistically significant relationship between the duration of cryopreservation and cell viability [20]. It has been previously reported that hydroxyethyl starch (HES) can partially substitute DMSO, with a $6 \%$ HES plus 5\% DMSO solution showing a very slight increase in PBMC recovery [21]. In our study, only two out of six subjects showed PBMC post-thaw viability

Table I. Cytokine production capacity of fresh versus cryopreserved T cell subsets, after Phorbol-Myristate-Acetate/lonomycin/Monensin short-term activation.

\begin{tabular}{|c|c|c|c|c|c|c|}
\hline & \multicolumn{2}{|c|}{ IL-2 } & \multicolumn{2}{|c|}{ TNF- $\alpha$} & \multicolumn{2}{|c|}{ IFN- $\gamma$} \\
\hline & Fresh & Cryo & Fresh & Cryo & Fresh & Cryo \\
\hline \multirow[t]{2}{*}{$\mathrm{CD} 3+\mathrm{CD} 4+(\%)$} & $55.2(45.5-69.8)$ & $54.0(28.0-70.5)$ & $24.9(15.5-33.4)$ & $31.4(28.1-45.0)$ & $13.6(10.6-17.2)$ & $12.9(10.7-16.3)$ \\
\hline & \multicolumn{2}{|c|}{$p=0.56$} & \multicolumn{2}{|c|}{$p=0.031$} & \multicolumn{2}{|c|}{$p=0.84$} \\
\hline \multirow{2}{*}{ CD3+CD4- (\%) } & $21.8(18.9-43.5)$ & $36.5(8.4-44.1)$ & $18.0(14.6-24)$ & $22.7(16.4-33.1)$ & $21.2(13.5-30.4)$ & $21.7(17.3-49.7)$ \\
\hline & \multicolumn{2}{|c|}{$p=0.84$} & \multicolumn{2}{|c|}{$p=0.031$} & \multicolumn{2}{|c|}{$p=0.31$} \\
\hline
\end{tabular}

A

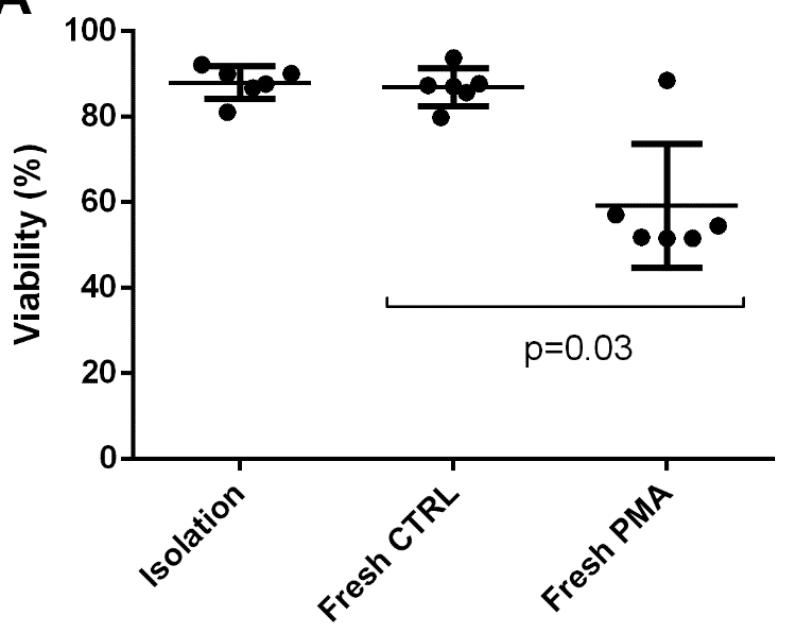

B

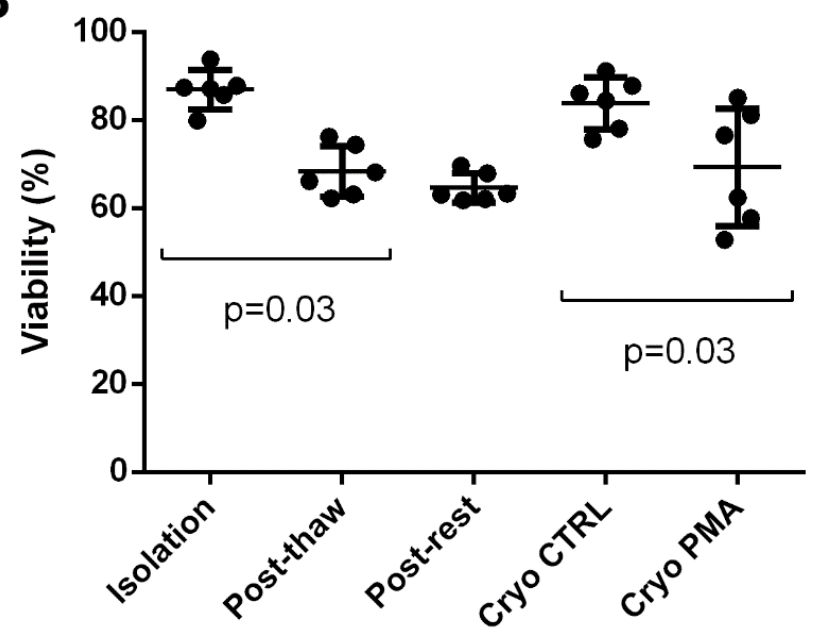

Fig. 2. Viability of (A) fresh and (B) cryopreserved PBMC after Ficoll separation and short-term activation with Phorbol-Myristate-Acetate/ lonomycin/Monensin. 
above $70 \%$, despite the use of optimal DMSO concentration.

Other findings suggest that the experience level of the laboratory staff members performing the cryopreservation is a major determinant of the viability of cryopreserved PBMC [22]. Maintenance of optimal storage conditions is also critical. It was shown that PBMC viability increases with decreasing temperatures [23]. In comparison to storage below $-135^{\circ} \mathrm{C}$, storage at $-80^{\circ} \mathrm{C}$ and above leads to progressive deterioration and loss of viability in both cells and tissues [24]. Significant deterioration in viability has been noted in PBMCs after 14 months of storage at $-80^{\circ} \mathrm{C}$ [16]. As such, this is another aspect which should be considered when developing pre-freeze and post-thaw viability assessment protocols. Incubating cells after thawing is a common practice when performing functional assays. We rested the cells post-thaw at $37^{\circ} \mathrm{C}$ for two hours in cRPMI in order to restore cell metabolism and functionality. Cell resting times are variable, allowing for practical variations in the laboratory set-up. It was shown that PBMCs can be incubated (rested) for up to 8 hours without affecting cell counts, but after 16 hours, viability is significantly decreased [25]. Lemieux showed that a rest of 1 hour was sufficient to restore T cells phenotype in thawed PBMCs [26].

Although PMA/ION is a very potent pharmacological cell stimulator, it is accepted that cell response to this stimulus represents the physiological cytokine production potential [27]. Studies showed that, upon 4-6hrs of stimulation, PMA/ION induces a greater cytokine response than other stimuli such as Phytohemagglutinin (PHA), Concanavalin A (ConA), and pokeweed mitogen (PWM) [28]. Nevertheless, if PMA/ION is used, cell loss is to be expected as it has been shown that not all cells survive throughout such an intense stimulation. However, since our aim was to investigate the cytokine-producing potential of PBMC in middle-aged subjects, we considered PMA/ION to be the most appropriate cell activator to use, despite its toxicity. As expected, and in agreement with a previous similar study performed in our laboratory [18], cell viability was significantly lower for stimulated cells compared to control unstimulated cells.

\section{Cytokine synthesis in fresh vs frozen T-cells}

In our study, we found that, after 5 hrs of stimulation with PMA/ION, cryopreserved CD4+ and CD4- T-cells produce TNF- $\alpha$ in greater proportions compared to freshly isolated and activated cells. This is important in clinical practice because oversecretion of TNF- $\alpha$ in frozen cells may have harmful effects when transplanted as was shown in rabbits where injection of TNF- $\alpha$ induces a shock-like state with increased vascular permeability, severe pulmonary edema, and hemorrhage [29].

TNF is a pleiotropic cytokine required for optimal defense against pathogens, proper lymphoid-organ architecture and germinal-center formation, development of granulomas, resolution of inflammation, and induction of tissue repair [30]. Once released, TNF- $\alpha$ and IL-1 act on different target cells, such as macrophages, endothelial cells, and neutrophils. TNF- $\alpha$ leads to an enhanced production of macrophages from progenitor cells [31], promotes the activation and differentiation of macrophages [32], and prolongs their survival [33]. All these effects enhance proinflammatory responses in sepsis. These cytokines act synergistically in the initiation of the inflammatory cascade of sepsis, resulting in hypotension, tachycardia, systemic edema, disseminated intravascular coagulation, and finally multiple organ system failure [34].

The multifaceted freezing and thawing process modulates the expression of thousands of genes, many involved in the cellular stress response, death and inflammation. It was shown that cryopreserved PBMCs have different gene expression patterns than their freshly isolated counterpart, with an increased expression of TNF and IFN- $\gamma$ genes alongside other IFN- $\gamma$ stimulated genes. These changes tend to be related to stress responses, immune activation and cell death, thus IFN- $\gamma$, TNF- $\alpha$ and associated cytokines are induced, promoting increased anti-microbial activities as part of an activated inflammatory response. Yang et al. used a lower concentration of DMSO (7.5\%) [15], which could be a reason for the different cytokine production pattern.

Regarding the production of IFN- $\gamma$ and IL-2, no significant difference was observed in our study between fresh and frozen cells. A recent study on stored PBMCs showed that cryopreservation can result in significant reduction of mean IFN- $\gamma$ production in TRAP megapool stimulated $\mathrm{CD} 4+$ cells with less significant loss of IFN- $\gamma$ production in CD8+ cells. In the same study, significant loss of IL-2 and TNF- $\alpha$ expression in CD4+ cells after cryopreservation was obtained, but there was no observed reduction in IL-2 or TNF- $\alpha$ production from CD8+ cells at either timepoint [35]. Given that the magnitude of the cytokine response is variable between stimulations with different agents, such differences are common between stimulation protocols.

Concerning the cytokine production in fresh T-cells, we found that IL-2 production in both CD4+ and CD4(considered mainly CD8+) cells was similar to a previous study conducted in our laboratory [18]. However, that same study reported a significantly higher proportion of TNF- $\alpha$ producing CD4+ cells which is the opposite of what we have observed in our study. The difference is most probably caused by the different protein transport inhibitors used in the two studies (Brefeldin A in the previous vs $\mathrm{MON}$ in the present study). It is known that MON and Brefeldin A display similar performances in stimulation protocols for IFN- $\gamma$ and IL-2 synthesis, but $\mathrm{MON}$ is a better suited protein transport inhibitor if the cytokine of interest is TNF- $\alpha$. The percentage of IFN- $\gamma$ producing cells (both CD4+ and CD4-) was higher in our study. One explanation could be the age of the subjects: $21-26$ in the last study and 40-50 in the present study. Some studies 
suggest IFN- $\gamma$ correlates positively with age [36,37], while others imply that there is a negative correlation for CD4+ T cells [38,39], but a positive one for CD8+ [39].

\section{Cryopreservation - advantages and challenges}

Cell cryopreservation has many advantages over the use of fresh samples, especially when it comes to studies conducted on large populations over extensive time periods. Processing the samples at multiple time points and/or locations throughout the study inevitably results in undesired variability. In order to minimize variability, cryopreserved samples collected throughout a study can easily be centralized and batch analyzed in one single laboratory at one particular time. Another advantage of cell cryopreservation is the future availability, as cells stay viable for extended periods of time, allowing for further analysis. Cell cryopreservation also poses some challenges such as the need for temperature-controlling devices to allow cells to cool gradually, the need for cryoprotective agents (CPA) and for standardized protocols and qualified personnel.

The main concern regarding cell cryopreservation is the formation of intra- and extracellular ice crystals which causes mechanical, chemical and osmotic cell damage. In order to preserve cell viability, CPAs must be used. The most commonly used CPA is dimethyl sulfoxide (DMSO) to which cell membranes are permeable. DMSO acts by preventing ice crystals formation and by reducing the electrolyte concentration in unfrozen solutions inside and outside the cell, therefore protecting the cells throughout the freeze-thaw cycles $[40,41]$. DMSO can be used as a CPA in concentrations ranging from $1 \%$ to $32 \%$ with an optimal concentration of approximately $10 \%$ [42]. In this study, PBMCs were cryopreserved in cRPMI medium with $10 \%$ DMSO and 20\% FBS. FBS also contributes to cell viability by adjusting the osmotic pressure and maintaining the integrity of cell membranes [43]. As a CPA, DMSO is less toxic and yields a higher rate of cell survivability to cryopreservation $[43,44]$.

Another important aspect for cell viability is the freezing/thawing rate. In order to have control over this aspect, special devices are required. Cooling the cells at a rate of $1^{\circ} \mathrm{C}$ per minute is recommended as it makes ice crystals formation less probable. In this study we used a FTS30 freezing container which provides the optimal cooling rate for cell cryopreservation. On the contrary, thawing the cells is a process best done rapidly in order to prevent cell damage caused by recrystallization [45]. In this study, the $1 \mathrm{~mL}$ cryovials containing cryopreserved cells were rapidly thawed (1-2 minutes) in a pre-heated water bath at $37^{\circ} \mathrm{C}$. Post-thaw exposure to DMSO was minimized by immediately washing the cells with cRPMI medium [46].

\section{Study limitations}

The empirical results reported herein should be considered with regard to some of the present study's limitations. Since ICS is a lengthy, costly and elaborate technique, our study was conducted on a limited number of subjects which is an issue to be addressed in the future. Although the phenotype $\mathrm{CD} 3+\mathrm{CD} 4$ - is sometimes used in practice as a substitute for $\mathrm{CD} 3+\mathrm{CD} 8+$ [47], the lack of $\mathrm{CD} 8$ surface staining in this study was another limitation as the response of cytotoxic $\mathrm{T}$ cells was not assessed directly.

\section{Conclusions}

Cryopreservation of Ficoll-isolated PBMCs for up to 3 months significantly increases the TNF- $\alpha$ production potential of short-term ex-vivo PMA/ION/MON activated T-cells in middle-aged clinically healthy subjects. We believe that this effect on TNF- $\alpha$ should be considered when employing cryopreserved PBMCs for downstream applications.

\section{Acknowledgments and funding}

The study was supported by internal funds of Advanced Medical and Pharmaceutical Research Center of "George Emil Palade" University of Medicine, Pharmacy, Science and Technology from Târgu-Mureș, Romania.

\section{Authors' contribution}

MV - Conceptualization, Data curation, Formal Analysis, Software, Writing - original draft

IMC - Conceptualization, Data curation, Formal Analysis, Software, Writing - original draft

IBM - Conceptualization, Data curation, Formal Analysis, Software, Writing - review \& editing

DRM - Data curation, Formal Analysis, Investigation, Software, Supervision, Validation, Visualization, Writing - review \& editing

MD - Conceptualization, Funding acquisition, Investigation, Methodology, Project administration, Resources, Software, Supervision, Validation, Visualization, Writing - review \& editing

\section{Conflict of interest}

None to declare

\section{References}

1. Cohen MC, Cohen S. Cytokine function: a study in biologic diversity. Am J Clin Pathol. 1996;105(5):589-598.

2. García Morán GA, Parra-Medina R, Cardona AG, et al. Cytokines, chemokines and growth factors. In: Anaya JM, Shoenfeld Y, RojasVillarraga $A$, et al., editors. Autoimmunity: From Bench to Bedside [Internet]. Bogota (Colombia): El Rosario University Press; 2013 Jul 18. Chapter 9.

3. Bienvenu J, Monneret G, Fabien N, Revillard JP. The clinical usefulness of the measurement of cytokines. Clin Chem Lab Med. 2000;38(4):267285.

4. Smith SG, Smits K, Joosten SA, et al. Intracellular Cytokine Staining and Flow Cytometry: Considerations for Application in Clinical Trials of Novel Tuberculosis Vaccines. PLoS One. 2015;10(9):e0138042.

5. Maecker HT, Rinfret A, D'Souza P, et al. Standardization of cytokine flow cytometry assays. BMC Immunol. 2005;6:13.

6. Jaimes MC, Maecker HT, Yan M, et al. Quality assurance of intracellular cytokine staining assays: analysis of multiple rounds of proficiency testing. J Immunol Methods. 2011;363(2):143-157.

7. De Rosa SC. Vaccine applications of flow cytometry. Methods. 2012;57(3):383-391. 
8. Shulman N, Bellew M, Snelling G, et al. Development of an automated analysis system for data from flow cytometric intracellular cytokine staining assays from clinical vaccine trials. Cytometry A. 2008;73(9):847856.

9. Goode I, Xu H, Ildstad ST. Regulatory B cells: the new "it" cell. Transplant Proc. 2014;46(1):3-8.

10. Zelle-Rieser $\mathrm{C}$, Thangavadivel S, Biedermann R, et al. T cells in multiple myeloma display features of exhaustion and senescence at the tumor site. J Hematol Oncol. 2016;9(1):116.

11. Bakhach J. The cryopreservation of composite tissues: Principles and recent advancement on cryopreservation of different type of tissues. Organogenesis. 2009;5(3):119-126.

12. Perdomo-Celis F, Salgado DM, Castañeda DM, Narváez CF. Viability and Functionality of Cryopreserved Peripheral Blood Mononuclear Cells in Pediatric Dengue. Clin Vaccine Immunol. 2016;23(5):417-426.

13. Dyer WB, Pett SL, Sullivan JS, et al. Substantial improvements in performance indicators achieved in a peripheral blood mononuclear cell cryopreservation quality assurance program using single donor samples. Clin Vaccine Immunol. 2007;14(1):52-59.

14. Disis ML, dela Rosa C, Goodell V, et al. Maximizing the retention of antigen specific lymphocyte function after cryopreservation. J Immunol Methods. 2006;308(1-2):13-18.

15. Yang J, Diaz N, Adelsberger J, et al. The effects of storage temperature on PBMC gene expression. BMC Immunol. 2016;17:6.

16. Kreher CR, Dittrich MT, Guerkov R, et al. CD4+ and CD8+ cells in cryopreserved human PBMC maintain full functionality in cytokine ELISPOT assays. J Immunol Methods. 2003;278(1-2):79-93.

17. Serban GM, Mănescu IB, Manu DR, Dobreanu M. Optimization of a density gradient centrifugation protocol for isolation of peripheral blood mononuclear cells. Acta Medica Marisiensis 2018;64(2):83-90.

18. Mănescu IB, Șerban GM, Manu DR, Dobreanu M. Variability of ex-vivo stimulated T-cells secretory profile in healthy subjects. Revista Romana de Medicina de Laborator. 2020;28(1), 75-89.

19. Wang SY, Hsu ML, Tzeng $\mathrm{CH}$, et al. The influence of cryopreservation on cytokine production by human $T$ lymphocytes. Cryobiology. 1998;37(1):22-29.

20. Kleeberger CA, Lyles $\mathrm{RH}$, Margolick JB, Rinaldo CR, et al. Viability and recovery of peripheral blood mononuclear cells cryopreserved for up to 12 years in a multicenter study. Clin Diagn Lab Immunol. 1999;6(1):1419.

21. Kenmochi T, Asano T, Maruyama M, et al. Cryopreservation of human pancreatic islets from non-heart-beating donors using hydroxyethyl starch and dimethyl sulfoxide as cryoprotectants. Cell Transplant. 2008;17(1-2):61-67.

22. Weinberg A, Zhang L, Brown D, et al. Viability and functional activity of cryopreserved mononuclear cells. Clinical and Diagnostic Laboratory Immunology. 2000 Jul;7(4):714-716.

23. Germann A, Oh YJ, Schmidt T, Schön U, et al. Temperature fluctuations during deep temperature cryopreservation reduce PBMC recovery, viability and T-cell function. Cryobiology. 2013;67(2):193-200.

24. Hunt CJ. Technical Considerations in the Freezing, Low-Temperature Storage and Thawing of Stem Cells for Cellular Therapies. Transfus Med Hemother. 2019;46(3):134-150.

25. Hønge BL, Petersen MS, Olesen R, et al. Optimizing recovery of frozen human peripheral blood mononuclear cells for flow cytometry. PLoS One. 2017;12(11):e0187440.

26. Lemieux J, Jobin C, Simard C, Néron S. A global look into human T cell subsets before and after cryopreservation using multiparametric flow cytometry and two-dimensional visualization analysis. J Immunol Methods. 2016;434:73-82.

27. Prussin C. Cytokine flow cytometry: understanding cytokine biology at the single-cell level. J Clin Immunol. 1997;17(3):195-204.

28. Ai W, Li H, Song $\mathrm{N}$, et al. Optimal method to stimulate cytokine production and its use in immunotoxicity assessment. Int J Environ Res Public Health. 2013;10(9):3834-3842.

29. Okusawa S, Gelfand JA, Ikejima T, et al. Interleukin 1 induces a shocklike state in rabbits. Synergism with tumor necrosis factor and the effect of cyclooxygenase inhibition. J Clin Invest. 1988;81(4):1162-1172.

30. Kalliolias GD, Ivashkiv LB. TNF biology, pathogenic mechanisms and emerging therapeutic strategies. Nat Rev Rheumatol. 2016;12(1):49-62.

31. Fahlman C, Jacobsen FW, Veiby OP, McNiece IK, et al. Tumor necrosis factor-alpha (TNF-alpha) potently enhances in vitro macrophage production from primitive murine hematopoietic progenitor cells in combination with stem cell factor and interleukin-7: novel stimulatory role of p55 TNF receptors. Blood. 1994;84(5):1528-1533.

32. Witsell AL, Schook LB. Tumor necrosis factor alpha is an autocrine growth regulator during macrophage differentiation. Proc Natl Acad Sci U S A. 1992;89(10):4754-8.

33. Conte D, Holcik M, Lefebvre CA, et al. Inhibitor of apoptosis protein clAP2 is essential for lipopolysaccharide-induced macrophage survival. Mol Cell Biol. 2006;26(2):699-708.

34. Hotchkiss RS, Moldawer LL, Opal SM, Reinhart K, Turnbull IR, Vincent JL. Sepsis and septic shock. Nat Rev Dis Primers. 2016;2:16045.

35. Ford T, Wenden C, Mbekeani A, et al. Cryopreservation-related loss of antigen-specific IFN $\gamma$ producing CD4+ T-cells can skew immunogenicity data in vaccine trials: Lessons from a malaria vaccine trial substudy. Vaccine. 2017;35(15):1898-1906.

36. Bandrés $E$, Merino J, Vázquez $B$, et al. The increase of IFN-gamma production through aging correlates with the expanded CD8(+high) CD28(-)CD57(+) subpopulation. Clin Immunol. 2000;96(3):230-235.

37. Sakata-Kaneko S, Wakatsuki Y, Matsunaga $Y$, et al. Altered Th1/Th2 commitment in human CD4+ $T$ cells with ageing. Clin Exp Immunol. 2000;120(2):267-273.

38. Alberti S, Cevenini E, Ostan R, et al. Age-dependent modifications of Type 1 and Type 2 cytokines within virgin and memory CD4+ T cells in humans. Mech Ageing Dev. 2006;127(6):560-566.

39. van der Geest KSM, Kroesen BJ, Horst G, Abdulahad WH, et al. Impact of Aging on the Frequency, Phenotype, and Function of CD161Expressing T Cells. Front Immunol. 2018;9:752.

40. Jang $\mathrm{TH}$, Park SC, Yang JH, et al. Cryopreservation and its clinical applications. Integr Med Res. 2017;6(1):12-18.

41. Ozkavukcu S, Erdemli E, Isik A, et al. Effects of cryopreservation on sperm parameters and ultrastructural morphology of human spermatozoa. J Assist Reprod Genet. 2008;25(8):403-411.

42. Hubálek Z. Protectants used in the cryopreservation of microorganisms. Cryobiology. 2003;46(3):205-229.

43. Yong KW, Pingguan-Murphy B, Xu F, et al. Phenotypic and functional characterization of long-term cryopreserved human adipose-derived stem cells. Sci Rep. 2015;5:9596.

44. Janz Fde L, Debes Ade A, Cavaglieri Rde C, et al. Evaluation of distinct freezing methods and cryoprotectants for human amniotic fluid stem cells cryopreservation. J Biomed Biotechnol. 2012;2012:649353.

45. Pegg DE. Principles of cryopreservation. Methods Mol Biol. 2007;368:39-57.

46. Fry LJ, Querol S, Gomez SG, McArdle S, et al. Assessing the toxic effects of DMSO on cord blood to determine exposure time limits and the optimum concentration for cryopreservation. Vox Sang. 2015;109(2):181-190.

47. Dunn HS, Haney DJ, Ghanekar SA, Stepick-Biek P, et al. Dynamics of CD4 and CD8 T cell responses to cytomegalovirus in healthy human donors. J Infect Dis. 2002;186(1):15-22. 